\title{
Microvascular Invasion Is an Independent Prognostic Factor in Patients with Prostate Cancer Treated with Radical Prostatectomy
}

\author{
Alberto A. Antunes, Miguel Srougi, Marcos F. Dall'Oglio, Alexandre Crippa, Mario Paranhos, \\ Jose Cury, Luciano J. Nesrallah, Katia R. Leite \\ Division of Urology, University of Sao Paulo Medical School and Laboratory of Surgical and \\ Molecular Pathology, Syrian Lebanese Hospital, Sao Paulo, Brazil
}

\begin{abstract}
Objective: Current published data regarding the prognostic value of microvascular invasion (MVI) in patients with prostate cancer ( $\mathrm{PCa}$ ) have yielded mixed results. Furthermore, most important series had surgical procedures performed by multiple surgeons and surgical specimens analyzed by multiple pathologists. We determined the relation of MVI with other pathologic features and whether this finding can be used as an independent prognostic factor in patients with PCa.

Materials and Methods: We selected 428 patients with clinically localized PCa treated with radical prostatectomy (RP). MVI was correlated to other pathologic features. The Kaplan-Meier method was used to evaluate survival curves and statistical significance was determined by the log-rank test. Multivariate analysis was performed through a Cox proportional hazards regression model.

Results: Eleven percent out of the 428 patients presented MVI. Except for the lack of association with biopsy Gleason score, MVI was related to all clinical and pathologic features of RP specimens. Mean follow up after surgery was $53.9 \pm$ 20.1 months. Patients with MVI presented a recurrence rate of $44.6 \%$ compared to only $20.2 \%$ for patients without MVI (Log-rank test - $\mathrm{p}<0.001$ ). After Cox regression analysis, MVI was an independent prognostic feature related to biochemical recurrence.

Conclusions: MVI is associated to advanced pathologic features of PCa and is an important prognostic factor regarding disease recurrence in patients treated with RP. These findings support the recommendations to the routine evaluation of this variable in pathologic reports of RP specimens.
\end{abstract}

Key words: prostate; prostatic neoplasms; prostatectomy; survival analysis; disease progression Int Braz J Urol. 2006; 32: 668-77

\section{INTRODUCTION}

Despite surgical treatment, almost $30 \%$ of the patients presenting clinically localized prostate cancer (PCa) will develop elevation of serum prostate specific antigen (PSA) in a 10 years follow up period (1). For this reason, one of the most important challenges for urologists is the identification of patients with a high risk of disease progression that may benefit from adjuvant therapies.

To date, histological characteristics of $\mathrm{PCa}$ in radical prostatectomy (RP) specimens as 
pathological stage, final Gleason score, seminal vesicle involvement, surgical margin status, level of extraprostatic extension and tumor volume are frequently used to predict progression after surgery (2). According to recommendations of the College of American Pathologists Cancer Committee since 1994, microvascular invasion (MVI) is reported in RP specimens (3), even though mixed results regarding its incidence and prognostic significance are reported in literature.

Incidence rates of MVI in RP specimens range from 5 to $53 \%$ (4-10). While many studies have found that MVI is a significant predictor of disease progression only in univariate analysis $(4,10)$, others have reported its independent significance in multivariate analysis $(8,9,11)$. Furthermore, the most important series had surgical procedures performed by multiple surgeons and surgical specimens analyzed by multiple pathologists, leading to possible interpretation biases.

In the present study, the authors sought to determine the prognostic significance of MVI in predicting biochemical recurrence after surgery in a group of patients with PCa treated with RP performed by a single surgeon and with surgical specimens analyzed by a single pathologist, enhancing the uniformity of MVI assessment and other pathologic variables.

\section{MATERIALS AND METHODS}

From August 1993 to November 2000, we selected 428 patients with PCa treated with RRP. Patients with insufficient clinical data or that had undergone neoadjuvant or adjuvant treatments were excluded from the study. All cases had clinically organ confined disease suspected by high serum PSA or palpable nodule at digital rectal examination and were diagnosed by transrectal ultrasound-guided needle biopsy. Surgical procedures were performed only by one surgeon (MS) and the pathology samples analyzed by only one pathologist (KRL). Staging evaluation consisted of history, physical examination, serum PSA, MVI, computed tomography, bone scan and TUNB. The clinical staging was determined using the
1992 AJCC staging system (12). Tumor grading was assessed according to the Gleason system (13).

The specimens of RP were submitted to histological study in accordance with the previously described recommendations (14). Thin transversal sections were performed in the surgical margins related to the bladder neck and the prostate apex. Seminal vesicles were sectioned in the base and longitudinal sections were submitted to histological examination. The entire gland was included for study after having their margins painted with India ink. Sequential transversal sections were performed every $3 \mathrm{~mm}$, designed from the proximal region towards the distal one. Approximately 10 or 12 sections from each lobe were included for histological study. The lymph nodes from the fat related to the resection of the iliac chain were dissected and included for study. The specimens of RP underwent the usual processing with inclusion in paraffin. MVI was defined as the presence of tumor cells within an endothelium lined spaces.

Mean patient age at diagnosis was 62.8 years (range 40 to 83 ), and mean PSA was $10.0 \mathrm{ng} / \mathrm{mL}$. Three hundred and thirty five $(78.2 \%)$ of the 428 patients presented Gleason score of six or less, and about $50 \%$ had non-palpable disease (T1c). The mean percent positive biopsy cores (PPBC) was $41 \%$ (range 5 to $100 \%$ ). After pathological examination, $72.1 \%$ of the patients showed organ confined disease (T2) and no patient showed lymph node involvement.

Patients were examined at month 2 postoperatively, and then every 6 months for 5 years and annually thereafter. At every follow up, digital rectal examination was performed and serum PSA measured. Disease recurrence was defined as a serum PSA of $0.4 \mathrm{ng} / \mathrm{mL}$ or greater.

To analyze the clinical and pathological variables according to the MVI, we used the Student's t-test, Fisher exact test and qui-squared test. PSA and PPBC were analyzed as continuous and categorical variables. To analyze the value of the pre-operative clinical and pathological parameters in predicting the presence of MVI at surgical specimen, we used a logistic regression model with adjusted proportional risks. Kaplan Meier method was used to estimate the survival curves and to compare the curves we used the 
log-rank test. A survival analysis, considering biochemical recurrence as the main end point was done through a Cox regression model. Statistical significance was set as $p \leq 0.05$. Statistical analysis was performed using the SPSS 12.0 for Windows software.

\section{RESULTS}

MVI was found in 47 (11\%) of the 428 patients (CI 95\% [8.2\% to 14.3\%]). Table-1 lists clinical and pathological characteristics according to the presence of MVI. Serum PSA, analyzed as a continuous or categorical variable showed statistical association with the presence of MVI. While only 5\% of patients with PSA under $4 \mathrm{ng} / \mathrm{mL}$ had MVI, this finding was observed in almost $30 \%$ of patients with PSA greater than 20 $\mathrm{ng} / \mathrm{mL}$. Mean PPBC among patients with MVI was
$53 \%$ vs. $39 \%$ in patients without MVI. Likewise, about $21 \%$ of patients with more than $50 \%$ positive biopsy cores had MVI and only $8 \%$ of patients with $50 \%$ or less positive cores had this finding $(\mathrm{p}=0.001)$. Regarding clinical stage, MVI rates were significantly higher among patients with $\mathrm{T} 2$ when compared to patients with non-palpable disease.

Table-2 lists postoperative pathological characteristics according to the presence of MVI. Regarding pathological stage, MVI was found in $20.2 \%$ of patients with T3 vs. $10.9 \%$ of the patients with T2B + T2C disease. Patients with T2A disease presented the lowest MVI rates. Patients with final Gleason score 8 or 9 presented $31.8 \%$ of MVI compared to only $5.8 \%$ among patients with final Gleason score between 4 and 7 .

In logistic regression analysis to determine the risk of MVI at surgical specimen, we found that

Table 1 - Preoperative variables according to the presence of microvascular invasion (MVI).

\begin{tabular}{lccc}
\hline & \multicolumn{2}{c}{ Microvascular Invasion } & \\
& Yes (N = 47) & $\mathbf{N}(\mathbf{3 8 1})$ & p Value \\
\hline Age (years) & $63.8 \pm 7.2$ & $62.7 \pm 7.5$ & $0.372^{*}$ \\
PSA (mean) & $10.6[8.6 ; 13.1]$ & $8[7.5 ; 8.5]$ & $0.004^{* *}$ \\
PSA & & & $0.006^{* * *}$ \\
$\leq 4.0$ & $2(5.0 \%)$ & $38(95.0 \%)$ & \\
4.1 to 10.0 & $21(8.3 \%)$ & $232(91.7 \%)$ & \\
10.1 to 20.0 & $15(14.7 \%)$ & $87(85.3 \%)$ & $0.169^{* * *}$ \\
$>20.0$ & $9(27.3 \%)$ & $24(72.7 \%)$ & \\
Gleason score & & & \\
$\leq 6$ & $33(10.8 \%)$ & $302(90.2 \%)$ & $0.001^{*}$ \\
7 & $8(12.5 \%)$ & $56(87.5 \%)$ & \\
$\geq 8$ & $6(20.7 \%)$ & $23(79.7 \%)$ & \\
PPBC & $52.9 \% \pm 28.0 \%$ & $39.3 \% \pm 23.0 \%$ & \\
PPBC & & & \\
$\leq 25.0 \%$ & $11(7.8 \%)$ & $130(92.2 \%)$ & $0.006^{* * * * *}$ \\
25.1 to $50.0 \%$ & $16(8.4 \%)$ & $174(91.6 \%)$ & \\
50.1 to $75.0 \%$ & $10(16.7 \%)$ & $50(83.3 \%)$ & \\
75.1 to $100.0 \%$ & $10(27.0 \%)$ & $27(73.0 \%)$ & \\
Clinical stage & & & \\
T1 & $14(6.5 \%)$ & $201(93.5 \%)$ & \\
T2 & $33(15.5 \%)$ & $180(84.5 \%)$ &
\end{tabular}

* Student's $t$ test; ** Log-transformed student's $t$ test; *** Fisher exact test generalized; **** Fisher exact test; PPBC $=$ percent of positive biopsy cores. 
Table 2 - Postoperative variables according to the presence of microvascular invasion.

\begin{tabular}{lcrc}
\hline & Yes $(\mathbf{N}=\mathbf{4 7})$ & $\mathbf{N}(\mathbf{3 8 1})$ & p Value \\
\hline Capsular involvement & & & $<0.001^{*}$ \\
Yes & $43(18.5 \%)$ & $189(81.5 \%)$ & \\
No & $4(2.0 \%)$ & $192(98.0 \%)$ & $0.030^{* *}$ \\
Seminal vesicle involvement & & & \\
Yes & $6(26.1 \%)$ & $17(73.9 \%)$ & $<0.001^{* * *}$ \\
No & $41(10.1 \%)$ & $364(89.9 \%)$ & \\
Pathological stage & & & \\
T2A & $3(2.4 \%)$ & $123(97.6 \%)$ & $<(86.3 \%)$ \\
T2B & $13(13.7 \%)$ & $81(92.0 \%)$ & \\
T2C & $7(8.0 \%)$ & $73(80.2 \%)$ & \\
T3A & $18(19.8 \%)$ & $4(80.0 \%)$ & \\
T3B & $1(20.0 \%)$ & $18(78.2 \%)$ & \\
T3C & $5(21.8 \%)$ & & \\
Gleason score & & & \\
4 to 6 & $12(4.7 \%)$ & $78(90.7 \%)$ & \\
7 & $8(9.3 \%)$ & $58(68.2 \%)$ & \\
8 to 9 & $27(31.8 \%)$ & $307(89.0 \%)$ & \\
Margin status & & $74(89.2 \%)$ & \\
Positive & $38(11.0 \%)$ & & \\
Negative & $9(10.8 \%)$ & & \\
\hline
\end{tabular}

* Qui-square; ** Fisher exact test; *** Fisher exact test generalized.

while serum PSA, PPBC and clinical stage showed statistical significance in univariate analysis, only serum PSA (OR- 6.59; CI 95\% [1.27 to 34.33]; $\mathrm{p}=$ 0.025 ) and clinical stage (OR- 2.31; CI 95\% [1.17 to 4.57]; $p=0.016$ ) remained significant variables on multivariate analysis. Biopsy Gleason score showed no relation with MVI risk.

Mean follow-up after surgery was $53.9 \pm 20.1$ months. Of the 428 patients, 98 (23\%) presented disease recurrence, six (1.4\%) developed clinical metastasis, and no one died of PCa. Using the log rank test, we found that patients with MVI had a $44.6 \%$ probability of disease recurrence vs. $20.2 \%$ among patients without MVI ( $\mathrm{p}<0.001)$ (Figure-1).

On univariate Cox proportional hazards regression analysis, all clinical and pathological characteristics but patient age and biopsy Gleason score were significantly associated with an increased risk of biochemical progression after surgery (Table3). However, on multivariate analysis, only serum PSA, clinical stage, capsular involvement, surgical

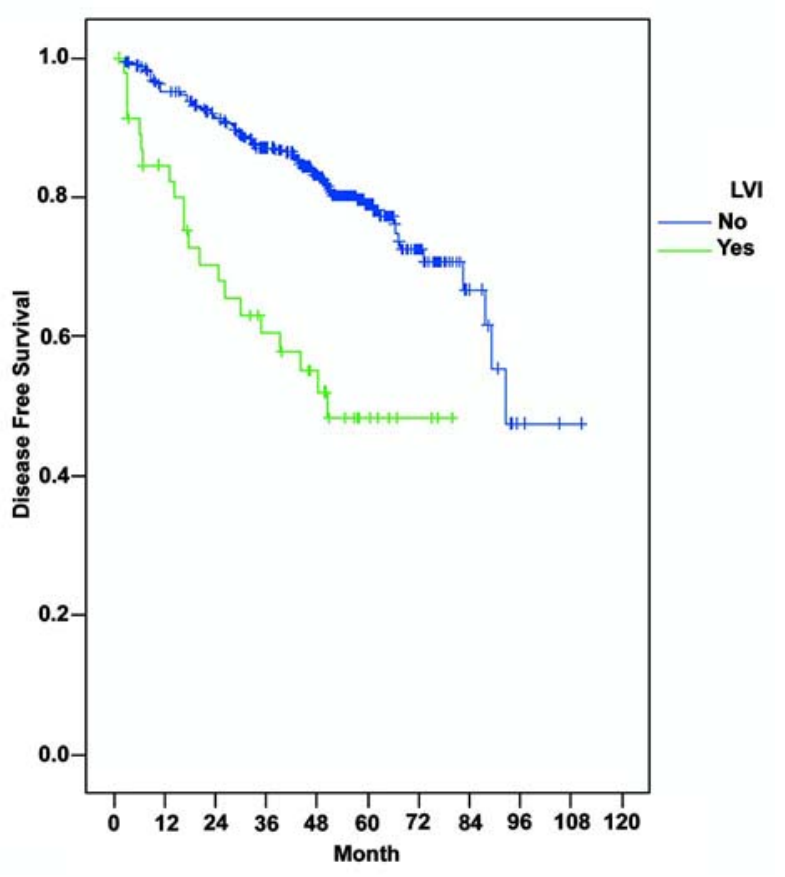

Figure 1 - Biochemical recurrence according to microvascular invasion (log-rank: $p<0.001$ ). 
Table 3-Cox regression for the risk of biochemical recurrence (univariate analysis).

\begin{tabular}{|c|c|c|c|}
\hline & Hazard Ratio & $95 \% \mathrm{CI}$ & p Value \\
\hline Age (years) & 1.03 & {$[1.00 ; 1.06]$} & 0.055 \\
\hline PSA & & & $<0.001$ \\
\hline$(4.1$ to 10.0$) /(0$ to 4.0$)$ & 8.57 & {$[1.18 ; 62.25]$} & 0.034 \\
\hline$(10.1$ to 20.0$) /(0$ to 4.0$)$ & 17.32 & {$[2.37 ; 126.78]$} & 0.005 \\
\hline$(>20.0) /(0$ to 4.0$)$ & 19.10 & {$[2.50 ; 146.13]$} & 0.005 \\
\hline PPBC & & & $<0.001$ \\
\hline$(25.1$ to $50 \%) /(<25 . \%)$ & 1.51 & {$[0.89 ; 2.58]$} & 0.129 \\
\hline$(50.1$ to $75 \%) /(<25 . \%)$ & 2.10 & {$[1.10 ; 4.00]$} & 0.025 \\
\hline$(75.1$ to $100 \%) /(<25 . \%)$ & 4.00 & {$[2.12 ; 7.54]$} & $<0.001$ \\
\hline Biopsy Gleason score & & & 0.073 \\
\hline $7 /(2$ to 6$)$ & 1.74 & {$[1.04 ; 2.89]$} & 0.034 \\
\hline$(8$ to 10$) /(2$ to 6$)$ & 1.55 & {$[0.74 ; 3.22]$} & 0.245 \\
\hline \multicolumn{4}{|l|}{ Clinical stage } \\
\hline$(\mathrm{T} 2 / \mathrm{T} 1)$ & 2.34 & {$[1.53 ; 3.57]$} & $<0.001$ \\
\hline MVI & & & \\
\hline$(\mathrm{Yes} / \mathrm{No})$ & 3.17 & {$[1.95 ; 5.16]$} & $<0.001$ \\
\hline \multicolumn{4}{|c|}{ Seminal vesicle involvement } \\
\hline Yes/No) & 2.99 & {$[1.69 ; 5.30]$} & $<0.001$ \\
\hline $\begin{array}{l}\text { Capsular involvement } \\
(\mathrm{Yes} / \mathrm{No})\end{array}$ & 3.17 & {$[1.99 ; 5.06]$} & $<0.001$ \\
\hline Pathological stage & & & $<0.001$ \\
\hline$(\mathrm{T} 2 \mathrm{~B}+\mathrm{T} 2 \mathrm{C}) /(\mathrm{T} 2 \mathrm{~A})$ & 2.50 & {$[1.32 ; 4.76]$} & 0.005 \\
\hline$(\mathrm{T} 3 \mathrm{~A}+\mathrm{T} 3 \mathrm{~B}+\mathrm{T} 3 \mathrm{C}) /(\mathrm{T} 2 \mathrm{~A})$ & 4.76 & {$[2.51 ; 9.02]$} & $<0.001$ \\
\hline Final Gleason score & & & 0.007 \\
\hline $7 /(2$ to 6$)$ & 1.44 & {$[0.85 ; 2.42]$} & 0.175 \\
\hline$(8$ to 10$) /(2$ to 6$)$ & 2.09 & {$[1.32 ; 3.33]$} & 0.002 \\
\hline $\begin{array}{l}\text { Margin status } \\
\text { (Positive/Negative) }\end{array}$ & 2.02 & {$[1.31 ; 3.10]$} & 0.001 \\
\hline
\end{tabular}

PPBC = percent of positive biopsy cores; $M V I=$ microvascular invasion.

margin status and the presence of MVI were significantly associated with biochemical recurrence (Table-4).

\section{COMMENTS}

In the present study, we found MVI in $11 \%$ of the 428 patients. This finding is similar to other reported series (9). However, discrepant rates have also been reported, and this fact is probably caused by different criteria in defining MVI, interobserver interpretation, specimen handling, and by patient selection. McNeal \& Yemoto (6), analyzing a similar group of patients with clinically localized disease found a $14 \%$ incidence rate, Herman (8), studying only patients with T3 tumors found a $35 \%$ rate, and Salomao et al. (5), found an overall incidence of 53\%. The lower incidence observed in our study could be explained by a selection bias since more than $70 \%$ of patients had organ confined disease and no one showed lymph node involvement. However, as surgical specimens were analyzed by a single pathologist, the uniformity of MVI assessment and 
Table 4 - Cox regression for the risk of biochemical recurrence (multivariate analysis).

\begin{tabular}{|c|c|c|c|}
\hline & Hazard Ratio & $95 \% \mathrm{CI}$ & p Value \\
\hline PSA & & & 0.010 \\
\hline$(4.1$ to 10.0$) /(0$ to 4.0$)$ & 8.77 & {$[1.18 ; 65.24]$} & 0.034 \\
\hline$(10.1$ to 20.0$) /(0$ to 4.0$)$ & 13.99 & {$[1.86 ; 105.00]$} & 0.010 \\
\hline$(>20.0) /(0$ to 4.0$)$ & 15.89 & {$[2.03 ; 124.54]$} & 0.008 \\
\hline PPBC & & & 0.085 \\
\hline$(25.1$ to $50 \%) /(<25 \%)$ & 1.15 & {$[0.66 ; 1.99]$} & 0.629 \\
\hline$(50.1$ to $75 \%) /(<25 \%)$ & 1.16 & {$[0.59 ; 2.29]$} & 0.664 \\
\hline$(75.1$ to $100 \%) /(<25 \%)$ & 2.23 & {$[1.15 ; 4.32]$} & 0.017 \\
\hline \multicolumn{4}{|l|}{ Clinical stage } \\
\hline$(\mathrm{T} 2 / \mathrm{T} 1)$ & 1.87 & {$[1.19 ; 2.95]$} & 0.007 \\
\hline MVI (Yes/No) & 1.78 & {$[1.06 ; 2.97]$} & 0.029 \\
\hline Capsular involvement (Yes/No) & 2.15 & {$[1.30 ; 3.57]$} & 0.003 \\
\hline Margin status (Positive/Negative) & 1.67 & {$[1.06 ; 2.62]$} & 0.028 \\
\hline
\end{tabular}

$P P B C=$ percent of positive biopsy cores MVI = microvascular invasion .

other pathologic variables support the reliability of our results.

We also confirmed the correlation between MVI and other clinical and pathological characteristics of PCa. Regarding preoperative variables, MVI was associated with an increasing PSA, increasing PPBC and with an advanced clinical stage. Capsular and seminal vesicle involvement, increasing Gleason score and an advanced pathological stage were also associated with MVI postoperatively,. Logistic regression analysis showed that patients with preoperative PSA greater than 20 $\mathrm{ng} / \mathrm{mL}$ or palpable (T2) disease had 6.5 and 2.3 times the risk of presenting MVI on surgical specimen respectively.

Regarding biochemical recurrence after RP, our study also supports MVI as an independent prognostic variable. This finding was reproduced by other authors. McNeal and Yemoto (6), analyzing 357 radical prostatectomy specimens, found that the only independent predictors of biochemical recurrence were MVI, carcinoma grade, and cancer volume. de la Taille (9), analyzing 241 patients, found that biochemical recurrence-free survival was $92.5 \%$ for the patients without MVI as compared to $30.1 \%$ for patients with MVI on prostate specimen examination.
MVI, preoperative PSA and pathological stage were independent prognostic variables of biochemical recurrence. Likewise, Ito et al. (11), found that MVI along with Gleason grade and capsular penetration, were disease recurrence independent prognostic factors. Herman et al. (8), found a significant correlation between MVI and increasing tumor volume, increasing Gleason grade, level of extraprostatic extension and the presence of seminal vesicle involvement. At 5 years, $45 \%$ and $21 \%$ of patients with and without MVI respectively presented disease progression. In multivariate analysis, MVI showed independent significance in predicting disease progression. More recently, Ferrari et al. (15), found a significant correlation with high Gleason grade, extracapsular extension, seminal vesicle invasion, increasing cancer volumes, positive margins, and elevated preoperative PSA levels. MVI was a strong and independent predictor for disease recurrence, however, in contrast to our study, the surgical procedures in this series were performed by several surgeons and it is possible that different surgical techniques have affected outcomes in the study group.

Conversely, to these reports, Bahnson et al. (4), described that despite the association of MVI with a fourfold greater incidence of progression and or 
death, in multivariate analysis, its prognostic significance was dependent upon tumor grade. More recently, Shariat et al. (10), confirmed the association of MVI with features of biologically aggressive PCa and found a strong association between MVI and metastasis to regional lymph nodes. These findings suggest that MVI precedes or occurs coincidently with lymph node metastasis, however MVI was not an independent prognostic factor for biochemical recurrence on multivariate analysis. These results could be attributed to the short follow up period (median 21 months). Again, multiple surgeons and pathologists were involved in this report, contributing to possible interpretation biases.

Some limitations in this study should be considered. Since no patient presented lymph node involvement, this variable could not be related to MVI or used in multivariate analysis. Furthermore, despite our mean 53.9 months follow-up being longer than most previous studies analyzing MVI, the number of patients who developed clinical metastasis was limited (only six), and no one died of PCa. This fact limits the power of our study to analyze associations between these outcomes and MVI. Finally, the number of MVI foci was not quantified on surgical specimens, and this information could have provided additional prognostic information since the finding of more than five foci seems to connote a dire prognosis (15).

In conclusion, with the present study, we support MVI as an important pathologic feature in a group of patients with PCa treated by the same surgeon and with pathological analysis performed by a single pathologist. After the follow-up period, $44.6 \%$ vs. $20.2 \%$ of the patients with and without MVI had biochemical recurrence respectively. Additionally, MVI along with serum PSA, clinical stage, capsular involvement and surgical margin status were independent predictors for recurrence when controlled for the other variables. This variable should be routinely used to help selection of patients to adjuvant treatments.

\section{ACKNOWLEDGEMENTS}

Adriana Sanudo performed statistical analysis and Rita Ortega edited the article.

\section{CONFLICT OF INTEREST}

\author{
None declared.
}

\section{REFERENCES}

1. Hull GW, Rabbani F, Abbas F, Wheeler TM, Kattan MW, Scardino PT: Cancer control with radical prostatectomy alone in 1,000 consecutive patients. J Urol. 2002; 167: 528-34.

2. Mazzucchelli R, Santinelli A, Lopez-Beltran A, Scarpelli M, Montironi R: Evaluation of prognostic factors in radical prostatectomy specimens with cancer. Urol Int. 2002; 68: 209-15.

3. Henson DE, Hutter RV, Farrow G: Practice protocol for the examination of specimens removed from patients with carcinoma of the prostate gland. A publication of the cancer committee, college of American pathologists. Task Force on the Examination of Specimens Removed From Patients With Prostate Cancer. Arch Pathol Lab Med. 1994; 118: 779-83.

4. Bahnson RR, Dresner SM, Gooding W, Becich MJ: Incidence and prognostic significance of lymphatic and vascular invasion in radical prostatectomy specimens. Prostate. 1989; 15: 149-55.

5. Salomao DR, Graham SD, Bostwick DG: Microvascular invasion in prostate cancer correlates with pathologic stage. Arch Pathol Lab Med. 1995; 119: 1050-4.

6. McNeal JE, Yemoto CE: Significance of demonstrable vascular space invasion for the progression of prostatic adenocarcinoma. Am J Surg Pathol. 1996; 20: 135160 .

7. van den Ouden D, Kranse R, Hop WC, van der Kwast $\mathrm{TH}$, Schroder FH: Microvascular invasion in prostate cancer: prognostic significance in patients treated by radical prostatectomy for clinically localized carcinoma. Urol Int. 1998; 60: 17-24.

8. Herman CM, Wilcox GE, Kattan MW, Scardino PT, Wheeler TM: Lymphovascular invasion as a predictor of disease progression in prostate cancer. Am J Surg Pathol. 2000; 24: 859-63.

9. de la Taille A, Rubin MA, Buttyan R, Olsson CA, Bagiella E, Burchardt M, et al.: Is microvascular invasion on radical prostatectomy specimens a useful predictor of PSA recurrence for prostate cancer patients? Eur Urol. 2000; 38: 79-84.

10. Shariat SF, Khoddami SM, Saboorian H, Koeneman KS, Sagalowsky AI, Cadeddu JA, et al.: 
Lymphovascular invasion is a pathological feature of biologically aggressive disease in patients treated with radical prostatectomy. J Urol. 2004; 171: 1122-7.

11. Ito K, Nakashima J, Mukai M, Asakura H, Ohigashi T, Saito S, et al.: Prognostic implication of microvascular invasion in biochemical failure in patients treated with radical prostatectomy. Urol Int. 2003; 70: 297-302.

12. Beahrs OH, Henson DE, Hutter RVP: AJCC Manual for Staging of Cancer, 4th (ed.), Philadelphia, JB Lippincott. 1992.

13. Gleason DF: Histologic Grading and Clinical Staging of Prostatic Carcinoma. In: Tannenbaum M (ed.),
Urologic Pathology: The Prostate. Philadelphia, Lea \& Febiger. 1977; pp. 171-97

14. Bostwick DG, Foster CS: Examination if radical prostatectomy specimens: Therapeutic and prognostic significance. In: Foster W, Bostwick D (ed.), Pathology of Prostate, Series Major Problems in Pathology. Philadelphia, WB Saunders Co. 1998; vol. 34, p. 172.

15. Ferrari MK, McNeal JE, Malhotra SM, Brooks JD: Vascular invasion predicts recurrence after radical prostatectomy: stratification of risk based on pathologic variables. Urology. 2004; 64: 749-53.

Accepted after revision:

May 31, 2006

\author{
Correspondence address: \\ Dr. Miguel Srougi \\ Rua Barata Ribeiro, 414, 7o. andar \\ São Paulo, SP, 01308-000, Brazil \\ Fax: + $55113257-8002$ \\ E-mail: srougi@terra.com.br
}

\section{EDITORIAL COMMENT}

The authors from Sao Paulo present another manuscript on the age-old question: Does microvessel invasion (MVI) in the prostate offer any unique prognostic information? Is there any significance to the prostate cancer cell(s) that penetrate or abut the wall of vascular endothelial cells? Could this pathological finding lead to microscopic hematogenous dissemination of cells, leading to a biochemical recurrence, even in those patients with organ-confined disease?

The urologic literature contains numerous papers on this topic dating back at least 10 years
(McNeal et al.). There are noteworthy pitfalls when determining the true MVI within a particular radical prostatectomy specimen, such as the consistency of microvessel density (MVD) readings amongst individual pathologists and the quantitative ability to determine MVI. If this was standardized, the more relevant clinical question becomes whether MVI offers anything unique above the standard pathological and clinical parameters that are currently used to predict recurrence.

In this paper from Sao Paulo, the authors have evaluated 428 patients who had radical 
prostatectomies performed by a single surgeon with a mean follow-up of 53 months. The pathology was read by a single pathologist, and although this can be considered a strong point, the question of variability of the MVD readings was not addressed in this paper. Of all the cases examined, only $11 \%$ were found to have MVI. What if there were several pathologists reviewing this blindly. Would that $11 \%$ be consistent?

The significant findings of this paper were that MVI had a $44.6 \%$ probability of disease recurrence vs. $20.2 \%$ among patients without MVI ( $<<0.001)$. Unfortunately, there was no data presented in the paper indicating whether or not these biochemical recurrences were early or late when compared to the patients that did not have MVI. In addition, in an era when PSA doubling time has become an important surrogate prognostic indicator of metastatic disease and survival, there should have been some mention of this indicator. This type of information may have been meaningful, and should be explored, especially since there were

\section{EDITORIAL COMMENT}

The importance of microvascular invasion (MVI) on prognostic information in urological malignancy is fully evaluated in the recent years $(1,2)$. This paper presented the prognostic significance of MVI for biochemical recurrence after radical prostatectomy in 428 patients with prostate cancer. As the data were collected by a single surgeon and examined by a single pathologist, the analysis is consistent and reasonable. Multivariate analysis only 6 patients that developed metastatic disease in the study and there were no prostate cancer deaths. Interestingly, there was a lack of association with biopsy Gleason score, which has been determined by previous authors. So, are these findings meaningful, and will they change the patterns of care of our patients? Should this be done routinely, as the authors suggest, and discussed with patients in the postoperative consultation? "Sir, your have MVI, so, we now recommend...??"

Clearly, this needs more validation before we can use this to base adjuvant treatment decisions. I believe that we should be ordering tests only if we are going to act on the outcome. Otherwise, it is a research tool, and what is truly needed to answer this question is a randomized trial, which would demonstrate that adjuvant therapy in patients with MVI will improve the outcome, or have one as good as the MVI negative patients. Until then, its still research, and I commend the authors for trying to determine the true significance of this pathological finding (artifact?).

Dr. Aaron Edward Katz. Associate Professor of Clinical Urology College of Physicians of Surgeons, Columbia University New York, NY, USA E-mail:aek4@columbia.edu

demonstrated that 5 factors including serum PSA, clinical stage, capsular involvement, surgical margin status, and the presence of MVI were significant predictors for biochemical recurrence. As would be expected, the importance of MVI on prognostic impact for biochemical recurrence is not so high that other factors such as PSA and capsular involvement were still strong in this study population. For a given PSA value and/or presence or absence of capsular 
involvement, how can MVI add prognostic information to these already known poor prognostic factors? The re-evaluation for the importance of MVI in more homogenous subpopulation might be more informative. Furthermore, in a clinical point of view, risk stratification or nomogram to identify the patients who should receive adjuvant therapy would need to be created. We still have an effort to confirm the importance of MVI for prognostic information for prostate cancer in a large, randomized, prospective study.

\section{REFERENCES}

1. Kikuchi E, Horiguchi Y, Nakashima J, Hatakeyama N, Matsumoto M, Nishiyama T et al.: Lymphovascular invasion independently predicts increased disease specific survival in patients with transitional cell carcinoma of the upper urinary tract. J Urol. 2005; 174: 2120-3.

2. Quek ML, Stein JP, Nichols PW, Cai J, Miranda G, Groshen S, et al.: Prognostic significance of lymphovascular invasion of bladder cancer treated with radical cystectomy. J Urol. 2005: 174: 103-6.

Dr. Eiji Kikuchi \& Dr. Masaru Murai Department of Urology Keio University School of Medicine Tokyo, Japan E-mail:eiji-k@kb3.so-net.ne.jp 